\title{
SPECTRAL RESOLUTION OF THE IDENTITY FOR MATRICES OF ELEMENTS OF A LIE ALGEBRA
}

\author{
H. S. GREEN
}

(Received 21 April 1975)

\begin{abstract}
This is an application of the characteristic identity satisfied by matrices whose elements are also elements of a semi-simple Lie algebra. Generalized eigenvectors are determined for matrices consisting of generators of $G L(n)$, $O(n)$ and $S p(n)$, and it is shown how to resolve the identity into idempotents constructed from such eigenvectors. By this means rather general functions of the matrices may be defined. It is also shown how to determine traces of such functions, in terms of the invariants of the Lie algebra.
\end{abstract}

\section{Introduction}

The generators of various classical groups may be assembled to form a square matrix $a$, of which the elements $a_{p q}(p, q=1,2, \cdots n)$ are also elements of a semi-simple Lie algebra [1]. Although the elements of such a matrix do not commute with one another in general, it has many properties analogous to those of numerical matrices. Powers $a^{j}$ of the matrix can be defined in the usual way by the formulae

$$
\begin{aligned}
& \left(a^{0}\right)_{p q}=\delta_{p q} \\
& \left(a^{\prime}\right)_{p q}=a_{p r}\left(a^{j-1}\right)_{r q}
\end{aligned}
$$

(where repeated affixes like $p, q, r$ are considered to be summed from 1 to $n$ ). From these powers of $a$, polynomials in $a$ may also be constructed. If the generators are suitably chosen, the traces of arbitrary polynomials in $a$, in particular the

$$
\operatorname{tr}\left(a^{\prime}\right)=\left(a^{i}\right)_{p p}
$$

are invariants, i.e., commute with all elements of the Lie algebra. 
It is known from the work of Ilamed [2] that any matrix $a$ whose elements are also elements of an associative algebra must satisfy a characteristic identity, analogous to the Cayley-Hamilton identity satisfied by numerical matrices. Explicit identities of the type

$$
\prod_{k=1}^{n}\left(a-\alpha_{k}\right)=0
$$

have been derived by Bracken and Green [3] and Green [4] for matrices whose elements are generators of $O(n), S p(n), G L(n)$ and related groups; the 'eigenvalues' $\alpha_{k}$ were determined as invariant multiples of the identity matrix $a^{0}$, and it was shown how to relate the $\alpha_{k}$ to the invariants $\operatorname{tr}\left(a^{i}\right)$. The transpose $b$ of $a$, defined as usual by

$$
b_{p q}=a_{q p}
$$

naturally satisfies a similar identity

$$
\prod_{k=1}^{n}\left(b-\beta_{k}\right)=0,
$$

where the $\beta_{k}$ are also invariant multiples of $a^{0}$.

The matrix $a$ may be applied from the left to a vector $\psi$, with components $\psi_{p}(p=1,2, \cdots n)$ which are, in general, linear operators and do not necessarily commute with one another, with the $a_{p q}$, or indeed with the $\alpha_{k}$. The transpose $b$ may be applied to such a vector from the right. The vector $\psi$ may be resolved into right 'eigenvectors' of $a$, or left 'eigenvectors' of $b$, thus:

$$
\begin{gathered}
\psi=\sum_{i=1}^{n} f_{j} \psi=\sum_{j=1}^{n} \psi g_{j} \\
f_{l}=\prod_{k \neq i}\left(\frac{a-\alpha_{k}}{\alpha_{i}-\alpha_{k}}\right), \quad g_{i}=\prod_{k \neq j}\left(\frac{b-\beta_{k}}{\beta_{i}-\beta_{k}}\right)
\end{gathered}
$$

where, because of (3) and (5),

$$
\left(a-\alpha_{,}\right)(f, \psi)=\left(\psi g_{\jmath}\right)(b-\beta,)=0 .
$$

With a non-degenerate numerical matrix $a$, it is possible to achieve spectral resolutions of the identity of the types

$$
\begin{aligned}
a^{0} & =\sum_{j=1}^{n} u_{j} c_{j} v_{j} \\
& =\sum_{j=1}^{n} v_{j} d_{j} u_{j}
\end{aligned}
$$

where 


$$
u_{i}=f_{j} \psi, \quad v_{j}=g, \phi
$$

are eigenvectors of $a$ and its transpose $b$, respectively, and the $c_{j}$ and $d_{j}$ are numerical constants. Such resolutions are useful, because they allow the definition of rather general functions of $a$ and $b$, by means of the formulae

$$
\begin{aligned}
& \theta(a)=\sum_{j=1}^{n} \theta\left(\alpha_{j}\right) u_{j} c_{j} v_{j} \\
& \theta(b)=\sum_{j=1}^{n} \theta\left(\beta_{j}\right) v_{j} d_{j} u_{j} .
\end{aligned}
$$

In this paper, we shall investigate the generalization of results such as (8) and (10) when the elements $a_{p q}$ of $a$ are also elements of semi-simple Lie algebras. Of special interest is a decomposition in which the vectors $u$, and $v_{f}$ are in the enveloping algebra of the Lie algebra, and therefore commute with $\alpha_{k}$ and $\beta_{k}$.

\section{Resolution for $G L(n)$}

If the $a_{p q}$ are generators of $G L(n)$, (see [5])

$$
\left[a_{p q}, a_{r s}\right]=\delta_{r q} a_{p s}-\delta_{p s} a_{r q},
$$

where, as usual, $[A, B]$ means $A B-B A$. The generators of $U(n)$ satisfy similar commutation relations, the only restriction arising from the condition that the elements $b_{p q}$ of the transpose of $a$ must also be the hermitean conjugate of $a_{p q}$. In any event, the generators satisfy characteristic identities of the types (3) and (5), where, according to [4],

$$
\begin{aligned}
& \alpha_{k}=\lambda_{k}+n-k \\
& \beta_{k}=\lambda_{k}+1-k
\end{aligned}
$$

and the eigenvalues $l_{k}$ of the $\lambda_{k}$ serve to identify irreducible representations of $G L(n)$; in the tensor representations, these eigenvalues are integers such that $l_{1} \geqq l_{2} \geqq \cdots \geqq l_{n} \geqq 0$. Also, the traces $\operatorname{tr}\left(a^{\prime}\right)$ are given by relations

$$
\begin{aligned}
\operatorname{tr}(a) & =\Sigma_{k} \lambda_{k}, \\
\operatorname{tr}\left(a^{2}\right) & =\Sigma_{k} \lambda_{k}\left(\lambda_{k}+n+1-2 k\right),
\end{aligned}
$$

etc., which can be used if desired to define the $\lambda_{k}$ independently, in an algebraic extension of the enveloping algebra of the Lie algebra. An independent method of obtaining these relations will be outlined in the last section of this paper.

Let us introduce two vectors $\psi$ and $\chi$, whose components satisfy 


$$
\begin{aligned}
& {\left[a_{p q}, \psi_{r}\right]=\delta_{q_{r}} \psi_{p}} \\
& {\left[a_{p q}, \chi_{r}\right]=\delta_{q^{\prime}} \chi_{p} .}
\end{aligned}
$$

These vectors are not defined within a particular irreducible representation of $G L(n)$, since, as we shall soon have occasion to notice, they have components which change one irreducible representation to another. Vectors with the required properties can, however, be found among the generators of groups, such as $G L(n+1)$ and $G L(n+2)$, of which $G L(n)$ is a subgroup; for instance, we could take $\psi_{r}=a_{r n+1}$ and $\chi_{r}=a_{r n+2}$, and the commutation relations (11), extended for values $n+1$ and $n+2$ of $p, q, r$ and $s$, would then include (14). The vectors $\psi$ and $\chi$ may be resolved, as shown in (6), into right and left eigenvectors of $a$ and $b$ respectively; it is known from earlier work of the author [4] that

$$
\lambda_{k}\left(f_{i} \psi\right)=\left(f_{,} \psi\right)\left(\lambda_{k}+\delta_{j k}\right),
$$

and it follows from (13) that

$$
a(f, \psi)=(f, \psi)(b+n),
$$

so that $f, \psi$ is also a left eigenvector of $b$ :

$$
f_{j} \psi=\psi g \text {. }
$$

Now, it can be seen from (15) that the tensor

$$
T_{p q}=\left(\psi g_{1}\right)_{p}\left(f_{i} \chi\right)_{q} .
$$

increases the eigenvalue $l$, of $\lambda_{i}$ in an irreducible representation of $G L(n)$ by two units, leaving the other eigenvalues $l_{k}$ unchanged. This (see [6]) is well known to be a property of symmetric tensors only, and it follows that

$$
\psi_{r}\left[\left(g_{1}\right)_{r p}\left(f_{i}\right)_{q s}-\left(g_{1}\right)_{r q}\left(f_{i}\right)_{p s}\right] \chi_{s}=0 .
$$

We now notice that, because the vectors $\psi$ and $x$ are arbitrary, they may be dropped from the equation, leaving the identity

$$
\left(g_{\jmath}\right)_{r p}\left(f_{1}\right)_{q s}=\left(g_{1}\right)_{r q}\left(f_{j}\right)_{p s} \text {. }
$$

[For instance, to eliminate the factor $\psi_{r}$, we may set $\psi_{r}=a_{r n+1}$, and take the commutator of (18) with $a_{n+1}$, . If, instead of $\psi$ and $\chi$, we had introduced two adjoint vectors, satisfying

$$
\left[a_{p q}, \phi_{r}\right]=-\delta_{p r} \phi_{q}
$$

we should have obtained the similar result 


$$
\left(f_{1}\right)_{r}\left(g_{j}\right)_{q s}=\left(f_{1}\right)_{r q}\left(g_{j}\right)_{p s}
$$

with $f_{j}$ and $g_{j}$ interchanged.

The results (20) and (22) have important consequences. We consider, if necessary, a subspace in which

$$
c_{s}=\left(f_{i}\right)_{n n}, \quad d,=\left(g_{j}\right)_{n n}
$$

are non-singular, and define a set of vectors $u$, and $v$, with components

$$
\begin{aligned}
& u_{j p}=\left(f_{j}\right)_{p n} c_{1}^{-1} \\
& v_{j p}=\left(g_{l}\right)_{p n} d_{l}^{-1} .
\end{aligned}
$$

By setting $q=r=s=n$ in (20) and (22) and dividing by $c$, and $d$, from right and left, we obtain

$$
\begin{aligned}
& u_{j p}=d_{l}^{-1}\left(g_{\jmath}\right)_{n p}, \\
& v_{\jmath p}=c_{\prime}^{-1}\left(f_{l}\right)_{n p} .
\end{aligned}
$$

By setting $r=s=n$ in (20) and (22), and again dividing by $c_{l}$ and $d_{j}$ from right and left, we have also

$$
\left[u_{j p}, u_{i q}\right]=\left[v_{j p}, v_{i q}\right]=0 .
$$

Thus, the components of either of these vectors commute among themselves; as $u_{j n}=v_{j n}=1$, this is a non-trivial result only for $n \geqq 3$. Finally, by setting $r=p=n$ in (20) and (22), we have

$$
\begin{aligned}
& \left(f_{i}\right)_{q s}=u_{j q} c_{j} v_{j s} \\
& \left(g_{j}\right)_{q s}=v_{j q} d_{j} u_{j s} .
\end{aligned}
$$

It follows, with the help of (6), that

$$
\begin{aligned}
& \delta_{p q}=\Sigma_{j}\left(f_{i}\right)_{p q}=\Sigma_{j}\left(u_{j p} c, v_{j q}\right), \\
& \delta_{p q}=\Sigma,\left(g_{j}\right)_{p q}=\Sigma_{j}\left(v_{j p} d_{j} u_{j q}\right) .
\end{aligned}
$$

This is the required generalization of (8); clearly, $c_{j}$ and $d_{j}$ are no longer numerical constants, but linear operators which do not commute with the vectors $u$, and $v_{i}$; but $u$, and $v_{i}$ are eigenvectors of $a$ and $b$, so that the analogy is a good one. In particular, it is easy to deduce results of the form (10) when $\theta(a)$ is any polynomial function of $a$; and the results may be extended to transcendental functions $\theta(a)$, provided $\theta\left(\alpha_{i}\right)$ and $\theta\left(\beta_{i}\right)$ are defined for all admissible eigenvalues of $\alpha_{j}$ and $\beta_{i}$.

The argument leading to (28) involved vectors $\psi, \chi, \phi, \cdots$ which could not be constructed from generators of $G L(n)$, but the final results do not involve 
such vectors and can be verified independently. However, the verification is by no means trivial even in the simplest instances. Thus, for $n=2$, the formula

$$
\left(f_{1}\right)_{11}=u_{11} c_{1} v_{11}
$$

reduces to a generalized determinantal identity

$$
\left(a_{11}-\lambda_{2}\right)=a_{12}\left(a_{22}-\lambda_{2}\right)^{-1} a_{21},
$$

which is verifiable with the help of (11) and (13), but is hardly obvious.

\section{Extension to $O(n)$ and $S p(n)$}

We now extend the results of the previous section to matrices constructed from generators of $O(n)$ and $S p(n)$, and their pseudo-orthogonal and pseudo-symplectic analogues. As in [4], we introduce a metric tensor $g_{p q}$, which is either symmetric or antisymmetric:

$$
g_{p q}=\eta g_{q p},
$$

where $\eta=+1$ for $O(n)$ or -1 for $S p(n)$. We require the existence of a corresponding contravariant tensor $g^{p q}$, satisfying

$$
g^{p r} g_{r q}=g_{q r} g^{p p}=\delta_{q}^{p} ;
$$

this imposes the usual limitation to even values of $n$ for $S p(n)$. The elements of the matrix $a$ in this instance will be denoted by $a^{p}{ }_{4}$, and those of the 'transpose' by

$$
b_{q}^{p}=a_{q}^{p} .
$$

The commutation relations are

$$
\left[a_{q}^{p}, a_{s}^{r}\right]=\delta_{q}^{r} a_{s}^{p}-\delta_{s}^{p} a_{q}^{r}-g^{p p} g_{q} a_{s}^{t}+g_{s q} g^{p t} a_{r}^{r} .
$$

The characteristic identities can still be written in the form (3) and (5), where

$$
\begin{gathered}
\alpha_{k}=\lambda_{k}+n-\eta-k, \\
\grave{\beta}_{k}=\lambda_{k}+1-k=-\alpha_{n+1-k} .
\end{gathered}
$$

The relation between the $\alpha_{k}$ and $\beta_{k}$ follows from the formulae

$$
\begin{array}{ll}
\lambda_{k}+\lambda_{n+1-k}=\eta & k \neq \frac{1}{2}(n+1) \\
\lambda_{k}=h=\frac{1}{2}(n-1) & k=\frac{1}{2}(n+1)
\end{array}
$$


defining the $\lambda_{k}$ for $k \geqq \frac{1}{2}(n+1)$ in terms of those for $k<\frac{1}{2}(n+1)$. Here again, the $\lambda_{k}$ are invariants whose eigenvalues $l_{k}$, for $k<\frac{1}{2}(n+1)$, serve to label irreducible representations, and can be defined implicitly by formulae connecting them with the traces of the even powers of $a$, e.g.,

$$
\operatorname{tr}\left(a^{2}\right)=2 \sum_{k=1}^{h} \lambda_{k}\left(\lambda_{k}+n+1-\eta-2 k\right),
$$

where $h=\frac{1}{2} n$ when $n$ is even, but $h=\frac{1}{2}(n-1)$ when $n$ is odd.

We next introduce two vectors $\psi$ and $\chi$, with components which satisfy

$$
\begin{aligned}
& {\left[a_{q}^{p}, \psi^{\prime}\right]=\delta_{q}^{r} \psi^{p}-g^{p p} g_{q t} \psi^{\prime},} \\
& {\left[a_{q}^{p}, \chi^{r}\right]=\delta_{q}^{r} \chi^{p}-g^{p} g_{q t} \chi^{\prime} .}
\end{aligned}
$$

The corresponding adjoint vectors satisfy commutation relations of the type

$$
\left[a_{q}^{p}, \phi_{s}\right]=-\delta_{s}^{p} \phi_{q}+g_{s q} g^{p t} \phi_{r} .
$$

Again it follows from earlier work of Bracken and Green [3] and Green [4] that

$$
\lambda_{j}\left(f_{k} \psi\right)=\left(f_{k} \psi\right)\left(\lambda_{j}+\delta_{j k}\right)
$$

and from (20) it follows that

$$
a\left(f_{k} \psi\right)=\left(f_{k} \psi\right)(b+n-\eta)
$$

so that

$$
\left(a-\alpha_{k}\right)\left(f_{k} \psi\right)=\left(f_{k} \psi\right)\left(b-\beta_{k}\right)=0,
$$

and

$$
f_{k} \psi=\psi g_{k} .
$$

From this point, the argument leading to the result (8) parallels that leading from (17) to (28) in the last section. As the tensor $T_{p q}$ defined by (18) increases the eigenvalue $l$, of $\lambda_{j}$ by two units, when $j \neq \frac{1}{2}(n+1)$ it corresponds to a representation of $O(n)$ or $S p(n)$ labelled $(2,0, \cdots)$ and is necessarily symmetric. Thus $(20)$ and (22) are still valid, for $j \neq \frac{1}{2}(n+1)$, and we can proceed to deduce (24), (25), (26) and (27). A special derivation of (27) is evidently needed only for the 'idempotents' $f_{m}$ and $g_{m}$, where $m=h+1=$ $\frac{1}{2}(n+1)$, for $O(n)$ when $n$ is odd. For this purpose, let us define

$$
\begin{aligned}
& u_{m}^{q}=\varepsilon^{q r s \cdots x y} a_{r s} \cdots a_{x y} \\
& v_{m q}=\varepsilon_{q r s \cdots x y} a^{r s} \cdots a^{x y},
\end{aligned}
$$


where

$$
a_{r s}=g_{n} a_{s,}^{\prime}, \quad a^{r s}=a_{r}^{r} g^{i s},
$$

and $\varepsilon$ is the permutation symbol. Then, by a calculation similar to that given by Bracken and Green [3, Appendix A], we verify that

$$
\begin{aligned}
& a_{q}^{p} u_{m}^{q}=h u_{m}^{p}, \\
& v_{m q} a_{p}^{q}=h v_{m p} .
\end{aligned}
$$

Thus, $u_{m}$ and $v_{m}$ are the right and left eigenvectors of $a$, corresponding to the eigenvalue $\lambda_{m}=h$, and we need only define

$$
\begin{aligned}
& c_{m}=\left(v_{m q} u_{m}^{q}\right)^{-1} \\
& d_{m}=\left(u_{m}^{q} v_{m q}\right)^{-1}
\end{aligned}
$$

to secure the correct normalization of $f_{m}$ and $g_{m}$, as given by (27). It should be noticed that, unlike the other $c_{l}$ and $d_{j}, c_{m}$ and $d_{m}$ thus defined are invariants which commute with all elements of the Lie algebra.

As the formulae (27) are now established for all values of $j,(28)$ follows.

\section{Evaluation of traces}

We shall now apply the foregoing to evaluate the trace of any well defined function $\theta(a)$ of a matrix $a$ of the type considered in the previous two sections. Since

$$
\operatorname{tr}[\theta(a)]=\Sigma, \theta\left(\alpha_{1}\right) \operatorname{tr}\left(f_{i}\right)
$$

it is clear that our purpose will be accomplished by the evaluation of

$$
t_{j}=\operatorname{tr}\left(f_{t}\right) \text {. }
$$

We consider first the application to matrices consisting of generators of $G L(n)$. It is evident from $\delta_{p r} \delta_{r q}=\delta_{p q}$ and (28) that

$$
\begin{aligned}
& v_{j p} u_{j p}=c_{1}^{-1}, \\
& u_{j p} v_{j p}=d_{1}^{-1},
\end{aligned}
$$

and from (27) that

$$
t_{j}=u_{j p} c_{j} v_{j p}
$$

But as the $c_{j}$, do not, in general, commute with the vectors $u_{j}$ and $v_{j}$, there is some difficulty in the direct application of this formula. From the definition of $f$, in (6), however, it follows that 


$$
\begin{aligned}
& t_{j}=p_{1} /\left[\prod_{k \neq j}\left(\alpha_{k}-\alpha_{j}\right)\right], \\
& p_{j}=\operatorname{tr}\left[\prod_{k \neq j}\left(a-\alpha_{\jmath}\right)\right]
\end{aligned}
$$

where $p$, must reduce to a polynomial of the $(n-1)$-th degree in the $\alpha_{k}$.

The polynomial can in fact be determined completely by general considerations. First we note that, if $a_{p q}$ is replaced by $a_{p q}+c \delta_{p q}$, where $c$ is any numerical constant or invariant, the commutation relations (11) are unchanged; also, although the 'eigenvalues' $\alpha_{k}$ of $a$ are replaced by $\alpha_{k}+c$, the 'eigenvectors' are unchanged; thus, $f_{\text {, and } t}$ cannot depend on $c$. It follows that the polynomials $p_{j}$ can only depend on the differences $\alpha_{k}-\alpha_{i}$ of the 'eigenvalues' of $a$. Moreover, by symmetry, $p$, must remain unchanged when $\alpha_{k}$ and $\alpha_{l}$ are interchanged, provided $k \neq j$ and $l \neq j$. We now observe that, according to (12),

$$
\alpha_{k}=\lambda_{k}+n-k
$$

also the eigenvalues $l_{k}$ of the $\lambda_{k}$ must satisfy $l_{1} \geqq l_{2} \geqq \cdots \geqq l_{n}$ in tensor representations. It follows that, if $\psi$ is any vector, $f_{j} \psi$ must vanish when applied to a tensor eigenvector of the $\lambda_{k}$ such that $l_{j}=l_{j+1}$. Hence, the trace of $f_{l}$ must possess a factor $\left(\alpha_{1}-\alpha_{t+1}-1\right)$. Taking account of the requirements of symmetry already noticed, we must have

$$
p_{1}=c \prod_{k \neq J}\left(\alpha_{j}-\alpha_{k}-1\right)
$$

where $c$ is a numerical constant. From the condition

$$
\operatorname{tr}\left(a^{0}\right)=\Sigma_{j} p_{\jmath} /\left[\prod_{k \neq l}\left(\alpha_{i}-\alpha_{k}\right)\right]=n,
$$

we infer that $c=1$, so that

$$
t_{j}=\prod_{k \neq j}\left(\frac{\alpha_{1}-\alpha_{k}-1}{\alpha_{1}-\alpha_{k}}\right) .
$$

From this result, and the formula (44), the relations (13) are easily confirmed, and a general formula for $\operatorname{tr}\left(a^{k}\right)$ can be written down.

Next, we consider the application to matrices consisting of generators of $O(n)$ or $S p(n)$. Again the invariants $t$, are given by (48), and again $t$, can only depend on differences of the 'eigenvalues' $\alpha_{k}$ of $a$, given this time by

$$
\alpha_{k}=\lambda_{k}+n-\eta-k
$$


However, the $\lambda_{k}$ cannot be assigned arbitrarily, but must satisfy (34). We must consider separately even and odd values of $n$.

For even values of $n$, symmetry required that $p$, must remain unchanged when $\alpha_{k}$ and $\alpha_{l}$ are interchanged, except when $k$ or $l$ takes one of the values $j$ or $n+1-j$. The eigenvalues $l_{k}$ of the $\lambda_{k}$ must satisfy $l_{1} \geqq l_{2} \geqq \cdots \geqq\left|l_{h}\right| \geqq 0$, where $h=\frac{1}{2} n$, in tensor or spinor representations, and so, if $j \neq h, f_{j} \psi$ must vanish when applied to a tensor or spinor eigenvector of the $\lambda_{k}$ such that $l,=l_{j+1}$. This and the requirements of symmetry already noted determine $n-2$ factors of $p_{i}$, of the type $\left(\alpha_{1}-\alpha_{k}-1\right)$ where $k \neq j$ and $k \neq n+1-j$. The additional factor, $\left(\alpha_{j}-\alpha_{n+1-1}\right)$, cancels a corresponding factor in the denominator of $t$, in (48); it is required so that $t$, does not become singular in spinor representations. If we again determine the numerical factor with the help of (50), we obtain

$$
\begin{aligned}
t,= & \prod_{k \neq f}\left(\frac{\alpha_{1}-\alpha_{k}-1+\eta \delta_{, n+1-k}}{\alpha_{j}-\alpha_{k}}\right) \\
& (n \text { even). }
\end{aligned}
$$

For odd values of $n$, we are restricted to $O(n)$, and $\lambda$, has the fixed value $h=\frac{1}{2}(n-1)$ when $j=m=h+1$. To determine $p_{m}$, we note that the vector $f_{m}$ does not change the eigenvalue of any of the $\lambda_{k}$, so that the condition $l_{1} \geqq l_{2} \geqq \cdots \geqq l_{h} \geqq 0$ does not impose any restriction (on $p_{m}$ ). But $t_{m}$ must not be singular in any representation, so that $p_{m}$ must possess a factor $\alpha_{m}-\alpha_{k}$ corresponding to every factor in the denominator of $t_{m}$, which therefore reduces to a numerical constant. As $f_{m}$ is an 'idempotent' (satisfying the matrix identity $f_{m}^{2}=f_{m}$ ), with a unique 'eigenvector' $u_{m}$ satisfying $f_{m} u_{m}=u_{m}$,

$$
t_{m}=1 \quad\left[m=\frac{1}{2}(n+1)\right] .
$$

When $j \neq m$ and $j \neq h, f, \psi$ must vanish when applied to a tensor or spinor eigenvector of the $\lambda_{k}$ such that $l_{l}=l_{j+1}$, so that again $p_{l}$ must possess a factor $\left(\alpha,-\alpha_{j+1}-1\right)$. This and the requirements of symmetry determine $n-3$ factors of $p_{j}$. A factor $\left(\alpha_{J}-\alpha_{m}\right)$ is clearly needed to cancel a corresponding

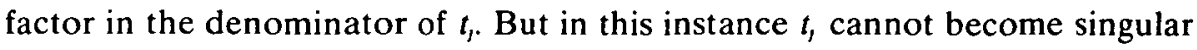
on account of the divisor $\left(\alpha_{1}-\alpha_{n+1-1}\right)$; the shift to a representation in which this divisor vanishes must be prevented by a factor $\left(\alpha_{j}-\alpha_{n+1-1}-2\right)$ in the numerator of $t$. Hence

$$
t_{j}=\prod_{k \neq 1}\left(\frac{\alpha_{L}-\alpha_{k}-1+\delta_{k m}-\delta_{\perp^{n+i-k}}}{\alpha_{1}-\alpha_{k}}\right)
$$


These results may be used, in conjunction with (44), to confirm the formula (35); of course, they may also be used to compute $\operatorname{tr}\left(a^{4}\right)$ and other traces which can only be determined with much greater labour by earlier methods.

\section{References}

[1] For a modern practical introduction to Lie algebras, see R. Gilmore, Lie Groups, Lie Algebras and Some of their Applications, (Wiley, New York, 1974).

[2] Y. (Lehrer-) Ilamed, Bull. Res. Comm. Israel 5A, 197 (1956).

[3] A. J. Bracken and H. S. Green, J. Math. Phys. 12, 2099 (1971).

[4] H. S. Green, J. Math. Phys. 12, 2106 (1971).

[5] M. Hammermesh, Group Theory, (Addison-Wesley, Reading, Mass., 1962), page 389.

[6] G. De B. Robinson, Representation Theory of the Symmetric Group (Edinburgh University Press, Edinburgh, 1961).

Department of Mathematical Physics, The University of Adelaide, Adelaide, S.A. 5000, Australia. 